\title{
A Regularity Criterion for Compressible Nematic Liquid Crystal Flows
}

\author{
Jishan Fan ${ }^{1}$ and Tohru Ozawa ${ }^{2}$ \\ ${ }^{1}$ Department of Applied Mathematics, Nanjing Forestry University, Nanjing 210037, China \\ ${ }^{2}$ Department of Applied Physics, Waseda University, Tokyo 169-8555, Japan
}

Correspondence should be addressed to Tohru Ozawa; txozawa@waseda.jp

Received 2 September 2013; Accepted 26 September 2013

Academic Editors: Y. Liu and X. B. Pan

Copyright (C) 2013 J. Fan and T. Ozawa. This is an open access article distributed under the Creative Commons Attribution License, which permits unrestricted use, distribution, and reproduction in any medium, provided the original work is properly cited.

We prove a blow-up criterion for local strong solutions to a simplified hydrodynamic flow modeling the compressible, nematic liquid crystal materials in a bounded domain.

\section{Introduction}

Let $\Omega \subset \mathbb{R}^{3}$ be a bounded domain with smooth boundary $\partial \Omega$. We consider the following simplified version of EricksenLeslie system modeling the hydrodynamic flow of compressible nematic liquid crystals:

$$
\begin{gathered}
\partial_{t} \rho+\operatorname{div}(\rho u)=0, \\
\partial_{t}(\rho u)+\operatorname{div}(\rho u \otimes u)+\nabla p(\rho)-\mu \Delta u \\
-(\lambda+\mu) \nabla \operatorname{div} u=-\Delta d \cdot \nabla d, \\
\partial_{t} d+u \cdot \nabla d=\Delta d+|\nabla d|^{2} d, \\
|d|=1 \quad \text { in } \Omega \times(0, \infty), \\
d=d_{0}(x) \quad \text { on } \partial \Omega \times(0, \infty), \\
(\rho, u, d)(x, 0)=\left(\rho_{0}, u_{0}, d_{0}\right)(x), \\
\left|d_{0}\right|=1, \quad x \in \Omega \subset \mathbb{R}^{3} .
\end{gathered}
$$

Here $\rho$ is the density of the fluid, $u$ is the fluid velocity, $d$ represents the macroscopic average of the nematic liquid crystal orientation field, and $p(\rho):=a \rho^{\gamma}$ is the pressure with positive constants $a>0$ and $\gamma>1$. Two real constants $\mu$ and $\lambda$ are the shear viscosity and the bulk viscosity coefficients of the fluid, respectively, which are assumed to satisfy the following physical condition:

$$
\mu>0, \quad 3 \lambda+2 \mu \geq 0 .
$$

Equations (1) and (2) are the well-known compressible Navier-Stokes system with the external force $-\Delta d \cdot \nabla d$. Equation (3) is the well-known heat flow of harmonic map when $u=0$.

Recently, Huang et al. [1] prove the following local-intime well-posedness.

Proposition 1. Let $\rho_{0} \in W^{1, q}$ for some $q \in(3,6]$ and $\rho_{0} \geq 0$ in $\Omega, u_{0} \in H^{2}, d_{0} \in H^{3}$ and $\left|d_{0}\right|=1$ in $\Omega$. If, in addition, the compatibility condition

$$
\begin{array}{r}
-\mu \Delta u_{0}-(\lambda+\mu) \nabla \operatorname{div} u_{0}-\nabla p\left(\rho_{0}\right)-\Delta d_{0} \cdot \nabla d_{0}=\sqrt{\rho_{0}} g \\
\text { for some } g \in L^{2}(\Omega)
\end{array}
$$

holds, then there exist $T_{0}>0$ and a unique strong solution $(\rho, u, d)$ to the problem $(1)-(5)$.

Based on the above proposition, Huang et al. [2] prove the regularity criterion

$$
\int_{0}^{T}\left(\|\mathscr{D}(u)\|_{L^{\infty}}+\|\nabla d\|_{L^{\infty}}^{2}\right) d t<\infty
$$

to the problem (1)-(3), (5) with the boundary condition

$$
u=0=\frac{\partial d}{\partial v} \quad \text { on } \partial \Omega \times(0, \infty)
$$

or

$$
u \cdot v=\operatorname{curl} u \times v=\frac{\partial d}{\partial \nu}=0 \quad \text { on } \partial \Omega \times(0, \infty) .
$$


Here,

$$
\mathscr{D}(u):=\frac{1}{2}\left(\nabla u+{ }^{t} \nabla u\right),
$$

where $\nu$ is the unit outward normal vector to $\partial \Omega$.

When $\Omega=\mathbb{R}^{3}$, Huang and Wang [3] show the following regularity criterion:

$$
\|\rho\|_{L^{\infty}\left(0, T ; L^{\infty}\right)}+\|u\|_{L^{s_{1}}\left(0, T ; L^{r_{1}}\right)}+\|\nabla d\|_{L^{s_{2}}\left(0, T ; L^{r_{2}}\right)}<\infty,
$$

with $r_{i}$ and $s_{i}$ satisfying

$$
\frac{2}{s_{i}}+\frac{3}{r_{i}}=1, \quad 3<r_{i} \leq \infty, i=1,2
$$

When the term $|\nabla d|^{2} d$ in (3) is replaced by $d-|d|^{2} d$, the problem (1)-(5) has been studied by L. M. Liu and X. G. Liu [4]; they proved the following regularity criterion:

$$
\int_{0}^{T}\left(\|\nabla u\|_{L^{2}}^{4}+\|\nabla u\|_{L^{\infty}}\right) d t<\infty
$$

The aim of this paper is to study the regularity criterion of local strong solutions to the problem (1)-(5). We will prove

Theorem 2. Let the assumptions in Proposition 1 hold true. If (12) holds true with $0<T<\infty$, then the solution $(\rho, u, d)$ can be extended beyond $T>0$.

Remark 3. Theorem 2 is also true for the boundary condition (9). But it is an open problem to prove (12) when the homogeneous Dirichlet boundary condition $u=0$ is replaced by

$$
u \cdot v=0, \quad \operatorname{curl} u \times v=0 \quad \text { on } \partial \Omega \times(0, \infty) .
$$

\section{Proof of Theorem 2}

Since $(\rho, u, d)$ is the local strong solution, we only need to prove a priori estimates.

First, testing (2) and (3) by $u$ and $\Delta d+|\nabla d|^{2} d$, respectively, and adding the resulting equations together, we see that

$$
\begin{aligned}
& \frac{d}{d t} \int\left(\frac{1}{2} \rho|u|^{2}+\frac{1}{2}|\nabla d|^{2}+\frac{a \rho^{\gamma}}{\gamma-1}\right) d x \\
& \quad+\int\left(\mu|\nabla u|^{2}+(\lambda+\mu)(\operatorname{div} u)^{2}+\left.\left.|\Delta d+| \nabla d\right|^{2} d\right|^{2}\right) d x=0,
\end{aligned}
$$

which gives

$$
\begin{aligned}
& \int\left(\rho|u|^{2}+|\nabla d|^{2}\right) d x \\
& \quad+\int_{0}^{T} \int\left(|\nabla u|^{2}+\left.\left.|\Delta d+| \nabla d\right|^{2} d\right|^{2}\right) d x d t \leq C .
\end{aligned}
$$

We decompose the velocity $u$ into two parts: $u=v+w$, where $v(t) \in H_{0}^{1}(\Omega) \cap H^{2}(\Omega)$ satisfies

$$
\mu \Delta v+(\lambda+\mu) \nabla \operatorname{div} v=\nabla p(\rho),
$$

and thus $w(t) \in H_{0}^{1}(\Omega) \cap H^{2}(\Omega)$ satisfies

$$
\mu \Delta w+(\lambda+\mu) \nabla \operatorname{div} w=\rho \dot{u}+\Delta d \cdot \nabla d,
$$

where we used $\dot{u}:=\partial_{t} u+u \cdot \nabla u$ to denote the material derivative of $u$. Then, together with the standard $W^{1, p}$ theory and $H^{2}$ theory for elliptic systems, we obtain

$$
\begin{gathered}
\|\nabla v\|_{L^{6}} \leq C\|p(\rho)\|_{L^{6}}, \\
\|\nabla w\|_{L^{6}}+\left\|\nabla^{2} w\right\|_{L^{2}} \leq C\|\rho \dot{u}\|_{L^{2}}+C\|\Delta d \nabla d\|_{L^{2}} .
\end{gathered}
$$

Testing (3) by $\Delta \partial_{t} d$ and using (4), (20), (3), and the identity $0=\Delta\left(d \partial_{t} d\right)=d \Delta \partial_{t} d+\partial_{t} d \Delta d+2 \nabla d \partial_{t} d$, we derive

$$
\begin{aligned}
& \frac{1}{2} \frac{d}{d t} \int|\Delta d|^{2} d x+\int\left|\nabla \partial_{t} d\right|^{2} d x \\
& =\int\left(u \cdot \nabla d-|\nabla d|^{2} d\right) \Delta \partial_{t} d d x \\
& =-\int \nabla(u \cdot \nabla d) \nabla \partial_{t} d d x-\int|\nabla d|^{2}\left(d \Delta \partial_{t} d\right) d x \\
& =-\int\left(u \nabla^{2} d+\nabla u \cdot \nabla d\right) \nabla \partial_{t} d d x \\
& +\int|\nabla d|^{2}\left(\partial_{t} d \Delta d+2 \nabla d \nabla \partial_{t} d\right) d x \\
& =-\int\left(u \cdot \nabla^{2} d+\nabla u \cdot \nabla d\right) \nabla \partial_{t} d d x \\
& +\int|\nabla d|^{2}\left(\Delta d+|\nabla d|^{2} d-u \cdot \nabla d\right) \Delta d d x \\
& +2 \int|\nabla d|^{2} \nabla d \nabla \partial_{t} d d x \\
& \leq\|u\|_{L^{r_{1}}}\left\|\nabla^{2} d\right\|_{L^{2 r_{1} /\left(r_{1}-2\right)}}\left\|\nabla \partial_{t} d\right\|_{L^{2}} \\
& +\|\nabla d\|_{L^{r_{2}}}\|\nabla u\|_{L^{2 r_{2} /\left(r_{2}-2\right)}}\left\|\nabla \partial_{t} d\right\|_{L^{2}} \\
& +C\|\nabla d\|_{L^{r_{2}}}^{2}\|\Delta d\|_{L^{2 r_{2} /\left(r_{2}-2\right)}}+C\|u\|_{L^{r_{1}}}^{2}\|\Delta d\|_{L^{r_{1} /\left(r_{1}-2\right)}}^{2} \\
& +\epsilon\left\|\nabla \partial_{t} d\right\|_{L^{2}}^{2} \\
& \leq C \epsilon\left\|\nabla \partial_{t} d\right\|_{L^{2}}^{2}+C\|u\|_{L^{r_{1}}}^{s_{1}}\left\|\nabla^{2} d\right\|_{L^{2}}^{2} \\
& +C\|\nabla d\|_{L^{r_{2}}}^{s_{2}}\|\nabla u\|_{L^{2}}^{2}+\epsilon\|\nabla u\|_{L^{6}}^{2} \\
& +C\|\nabla d\|_{L^{r^{2}}}^{s_{2}}\|\Delta d\|_{L^{2}}^{2}+\epsilon\|d\|_{H^{3}}^{2}+C \\
& \leq C \epsilon\left\|\nabla \partial_{t} d\right\|_{L^{2}}^{2}+C \epsilon\|d\|_{H^{3}}^{2}+C \epsilon\|\rho \dot{u}\|_{L^{2}}^{2} \\
& +C\|\nabla d\|_{L^{r_{2}}}^{s_{2}}\left(\|\Delta d\|_{L^{2}}^{2}+\|\nabla u\|_{L^{2}}^{2}\right) \\
& +C\|u\|_{L^{1_{1}}}^{s_{1}}\left\|\nabla^{2} d\right\|_{L^{2}}^{2}+C
\end{aligned}
$$

for any $0<\epsilon<1$, where we have used the Hölder inequality

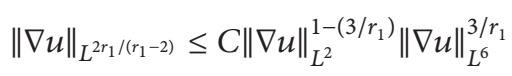


and the Gagliardo-Nirenberg inequality

$$
\begin{aligned}
\left\|\nabla^{2} d\right\|_{L^{2 r_{2} /\left(r_{2}-2\right)}} & \leq C\left\|\nabla^{2} d\right\|_{L^{2}}^{1-\left(3 / r_{2}\right)}\|d\|_{H^{3}}^{3 / r_{2}}, \\
\|\nabla u\|_{L^{6}} & \leq\|\nabla v\|_{L^{6}}+\|\nabla w\|_{L^{6}} \\
& \leq C+\|\nabla w\|_{L^{6}} .
\end{aligned}
$$

By the $H^{3}$ theory of the elliptic equations, it follows from (3) that

$$
\begin{aligned}
\|d\|_{H^{3}} \leq C(1 & \left.+\|\nabla \Delta d\|_{L^{2}}\right) \\
\leq & C\left(1+\left\|\nabla\left(\partial_{t} d+u \cdot \nabla d-|\nabla d|^{2} d\right)\right\|_{L^{2}}\right) \\
\leq & C\left(1+\left\|\nabla \partial_{t} d\right\|_{L^{2}}+\|u\|_{L^{r_{1}}}\left\|\nabla^{2} d\right\|_{L^{2 r_{1} /\left(r_{1}-2\right)}}\right. \\
& \left.+\|\nabla d\|_{L^{r_{2}}}\|\nabla u\|_{L^{2 r_{2} /\left(r_{2}-2\right)}}+\|\nabla d\|_{L^{r_{2}}}\left\|\nabla^{2} d\right\|_{L^{2 r_{2} /\left(r_{2}-2\right)}}\right) \\
\leq C(1 & +\left\|\nabla \partial_{t} d\right\|_{L^{2}}+\epsilon\|d\|_{H^{3}} \\
& +\|u\|_{L^{r_{1}}}^{s_{1} / 2}\left\|\nabla^{2} d\right\|_{L^{2}}+\epsilon\|\nabla u\|_{L^{6}} \\
& \left.+\|\nabla d\|_{L^{r_{2}}}^{s_{2} / 2}\|\nabla u\|_{L^{2}}+\|\nabla d\|_{L^{r_{2}}}^{s_{2} / 2}\|\Delta d\|_{L^{2}}\right),
\end{aligned}
$$

which yields

$$
\begin{aligned}
\|d\|_{H^{3}} \leq C(1 & +\left\|\nabla \partial_{t} d\right\|_{L^{2}}+\|u\|_{L^{r_{1}}}^{s_{1} / 2}\left\|\nabla^{2} d\right\|_{L^{2}} \\
& \left.+\|\nabla d\|_{L^{r_{2}}}^{s_{2} / 2}\|\nabla u\|_{L^{2}}+\|\nabla d\|_{L^{r_{2}}}^{s_{2} / 2}\left\|\nabla^{2} d\right\|_{L^{2}}\right) .
\end{aligned}
$$

Testing (2) by $\partial_{t} u$ and setting $M(d):=\nabla d \odot \nabla d-$ $(1 / 2)|\nabla d|^{2} \rrbracket_{3}$, we find that

$$
\begin{aligned}
\frac{1}{2} \frac{d}{d t} \int & \left(\mu|\nabla u|^{2}+(\lambda+\mu)(\operatorname{div} u)^{2}\right) d x+\int \rho|\dot{u}|^{2} d x \\
& -\frac{d}{d t} \int p \operatorname{div} u d x-\frac{d}{d t} \int M(d): \nabla u d x \\
= & \int \rho \dot{u} \cdot(u \cdot \nabla u) d x-\int \partial_{t} p \operatorname{div} u d x \\
& -\int \partial_{t} M(d): \nabla u d x \\
\leq & \|\rho \dot{u}\|_{L^{2}}\|u\|_{L^{r_{1}}}\|\nabla u\|_{L^{2 r_{1} /\left(r_{1}-2\right)}} \\
& +C\|\nabla d\|_{L^{r_{2}}}\|\nabla u\|_{L^{2 r_{2} /\left(r_{2}-2\right)}}\left\|\nabla \partial_{t} d\right\|_{L^{2}}-\int p_{t} \operatorname{div} u d x
\end{aligned}
$$

Now we deal with the last term.

First, (1) implies that

$$
\partial_{t} p+\operatorname{div}(p u)+(\gamma-1) p \operatorname{div} u=0 .
$$

Using (27) and (20), we have

$-\int \partial_{t} p \operatorname{div} u d x=-\int \partial_{t} p \operatorname{div} v d x-\int \partial_{t} p \operatorname{div} w d x$ $=\int \nabla \partial_{t} p v d x+\int \operatorname{div}(p u) \operatorname{div} w d x$ $+(\gamma-1) \int p \operatorname{div} u \operatorname{div} w d x$ $=-\frac{d}{d t} \int\left(\frac{\mu}{2}|\nabla v|^{2}+\frac{\lambda+\mu}{2}(\operatorname{div} v)^{2}\right) d x$ $-\int p u \nabla \operatorname{div} w d x$ $+(\gamma-1) \int p \operatorname{div} u \operatorname{div} w d x$ $\leq-\frac{d}{d t} \int\left(\frac{\mu}{2}|\nabla u|^{2}+\frac{\lambda+\mu}{2}(\operatorname{div} v)^{2}\right) d x$

$+C\|\sqrt{\rho} u\|_{L^{2}}\|\nabla \operatorname{div} w\|_{L^{2}}$

$+C\|\operatorname{div} u\|_{L^{2}}\|\operatorname{div} w\|_{L^{2}}$.

Inserting (28) into (26) and using (20), we have

$$
\begin{aligned}
\frac{1}{2} \frac{d}{d t} \int( & \left.\mu|\nabla u|^{2}+(\lambda+\mu)(\operatorname{div} u)^{2}\right) d x \\
& +\frac{d}{d t} \int\left(\frac{\mu}{2}|\nabla v|^{2}+\frac{\lambda+\mu}{2}(\operatorname{div} v)^{2}\right) d x \\
& -\frac{d}{d t} \int p \operatorname{div} u d x-\frac{d}{d t} \int M(d): \nabla u d x \\
& +\int \rho|\dot{u}|^{2} d x \\
\leq & \|\rho \dot{u}\|_{L^{2}}\|u\|_{L^{r_{1}}}\|\nabla u\|_{L^{2 r_{1} /\left(r_{1}-2\right)}} \\
& +C\|\nabla d\|_{L^{r_{2}}}\|\nabla u\|_{L^{2 r_{2} /\left(r_{2}-2\right)}}\left\|\nabla \partial_{t} d\right\|_{L^{2}} \\
& +C\|\nabla \operatorname{div} w\|_{L^{2}}+C\|\operatorname{div} u\|_{L^{2}}^{2}+C \\
\leq & \left.\epsilon \rho \dot{u}\right|^{2} d x+C\|u\|_{L^{r_{1}}}^{s_{1}}\|\nabla u\|_{L^{2}}^{2}+\epsilon\|\nabla u\|_{L^{6}}^{2} \\
& +C\|\nabla d\|_{L^{r_{2}}}^{s_{2}}\|\nabla u\|_{L^{2}}^{2}+\epsilon\left\|\nabla \partial_{t} d\right\|_{L^{2}}^{2} \\
& +C\|\nabla d\|_{L^{r_{2}}}^{s_{2}}\|\Delta d\|_{L^{2}}^{2}+C\|\operatorname{div} u\|_{L^{2}}^{2}+C \\
\leq & C \epsilon \int \rho|\dot{u}|^{2} d x+C \epsilon\left\|\nabla \partial_{t} d\right\|_{L^{2}}^{2}+C\|u\|_{L^{r_{1}}}^{s_{1}}\|\nabla u\|_{L^{2}}^{2} \\
& +C\|\nabla d\|_{L^{r_{2}}}^{s_{2}}\left(\|\nabla u\|_{L^{2}}^{2}+\|\Delta d\|_{L^{2}}^{2}\right)+C
\end{aligned}
$$

Combining (21), (25), and (29), taking $\epsilon$ small enough, and using the Gronwall inequality, we conclude that

$$
\begin{aligned}
& \|u\|_{L^{\infty}\left(0, T ; H^{1}\right)}+\|d\|_{L^{\infty}\left(0, T ; H^{2}\right)}+\|d\|_{L^{2}\left(0, T ; H^{3}\right)} \\
& \quad+\|\sqrt{\rho} \dot{u}\|_{L^{2}\left(0, T ; L^{2}\right)} \leq C .
\end{aligned}
$$


Now by the same calculations as those in $[3,5]$, we prove that

$$
\begin{gathered}
\|\rho\|_{L^{\infty}\left(0, T ; W^{1, q}\right)}+\left\|\partial_{t} \rho\right\|_{L^{\infty}\left(0, T ; L^{q}\right)} \leq C \\
\left\|\sqrt{\rho} \partial_{t} u\right\|_{L^{\infty}\left(0, T ; L^{2}\right)}+\left\|\partial_{t} u\right\|_{L^{2}\left(0, T ; H^{1}\right)} \leq C \\
\|u\|_{L^{\infty}\left(0, T ; H^{2}\right)}+\|u\|_{L^{2}\left(0, T ; W^{2, q}\right)} \leq C \\
\|d\|_{L^{\infty}\left(0, T ; H^{3}\right)} \leq C \\
\left\|\partial_{t} d\right\|_{L^{\infty}\left(0, T ; H^{1}\right)} \leq C
\end{gathered}
$$

This completes the proof.

\section{Acknowledgment}

This work is partially supported by NSFC (no. 11171154).

\section{References}

[1] T. Huang, C. Y. Wang, and H. Y. Wen, "Strong solutions of the compressible nematic liquid crystal flow," Journal of Differential Equations, vol. 252, no. 3, pp. 2222-2265, 2012.

[2] T. Huang, C. Y. Wang, and H. Y. Wen, "Blow up criterion for compressible nematic liquid crystal flows in dimension three," Archive for Rational Mechanics and Analysis, vol. 204, no. 1, pp. 285-311, 2012.

[3] X. Huang and Y. Wang, "A Serrin criterion for compressible nematic liquid crystal flows," Mathematical Methods in the Applied Sciences, vol. 36, no. 11, pp. 1363-1375, 2013.

[4] L. M. Liu and X. G. Liu, "A blow-up criterion for strong solutions to the compressible liquid crystals system," Chinese Annals of Mathematics A, vol. 32, no. 4, pp. 393-406, 2011.

[5] X. Huang and J. Lin, "Serrin-type blowup criterion for viscous, compressible, and heat conducting Navier-Stokes and magnetohydrodynamic flows," Communications in Mathematical Physics, vol. 324, no. 1, pp. 147-171, 2013. 


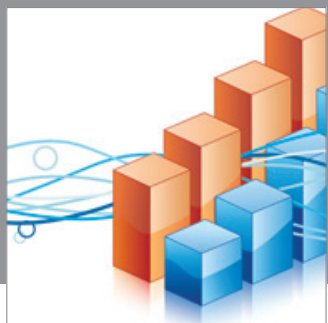

Advances in

Operations Research

mansans

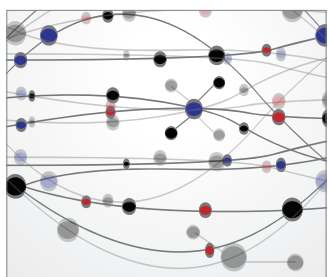

The Scientific World Journal
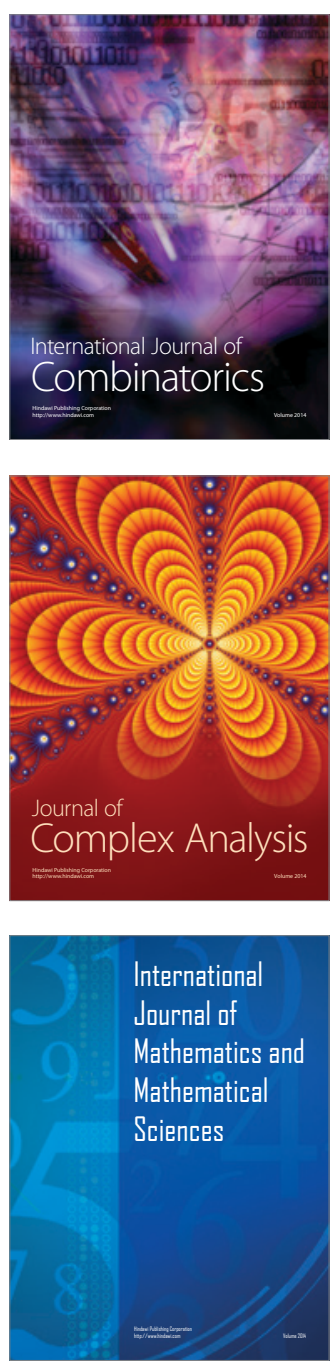
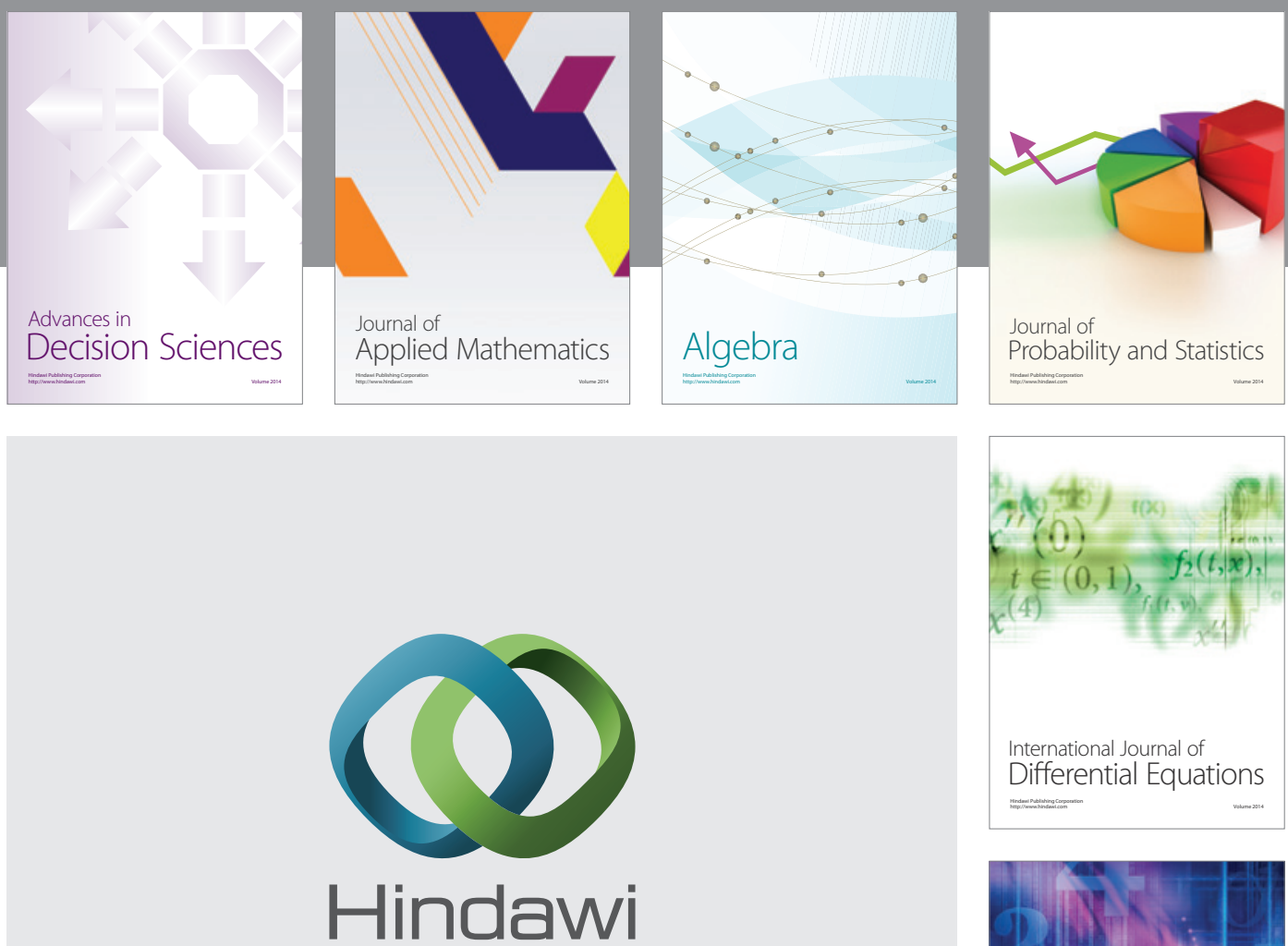

Submit your manuscripts at http://www.hindawi.com
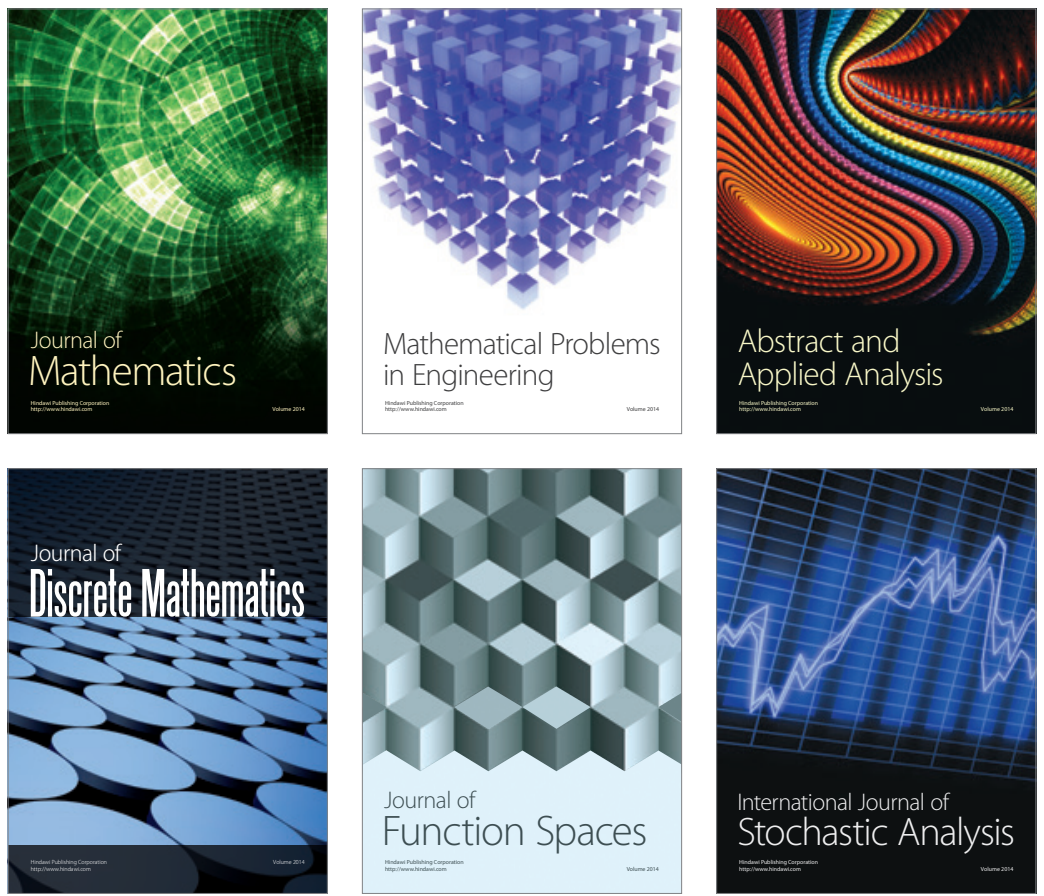

Journal of

Function Spaces

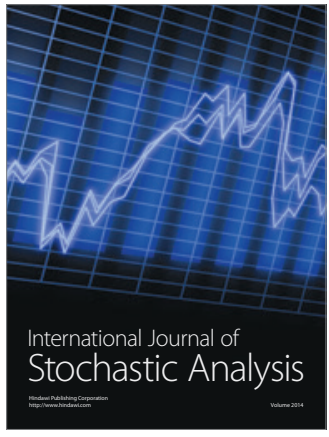

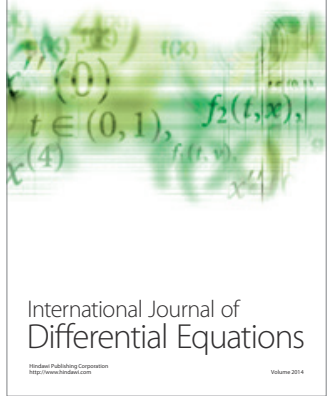
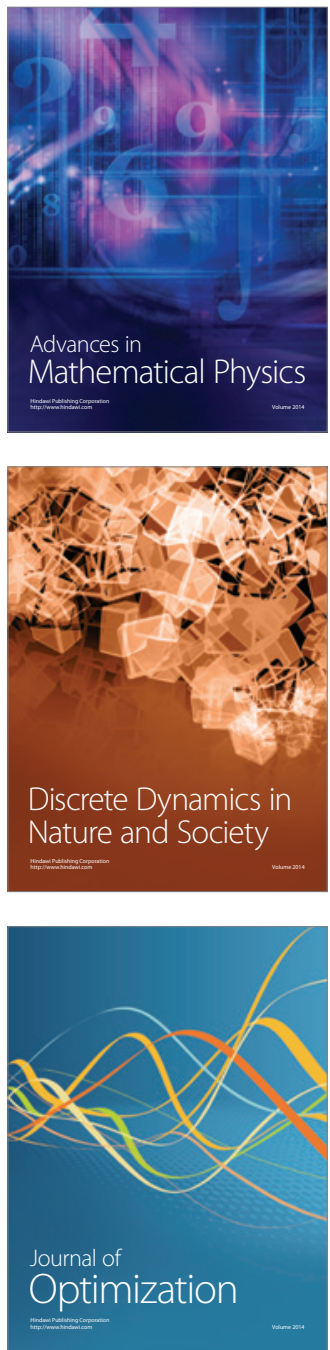